\title{
Structured Digital Storytelling for Eliciting Software Requirements in the ICT4D Domain
}

\author{
Daniel Sinnig ${ }^{1}$, Kristina Pitula ${ }^{1}$, Richard Becker ${ }^{2}$, \\ T. Radhakrishnan ${ }^{1}$, and Peter Forbrig ${ }^{2}$ \\ ${ }^{1}$ Concordia University, Montreal, Canada \\ \{d_sinnig,pitul_87,krishnan\}@encs.concordia.ca \\ ${ }^{2}$ University of Rostock, Germany \\ \{richard.becker, peter. forbrig\}@uni-rostock. de
}

\begin{abstract}
Due to the specific challenges that ICT4D projects present, conventional requirements elicitation techniques are often inapplicable or insufficient. We address this shortcoming by introducing structured digital storytelling (SDS) as an alternative elicitation technique especially suited for the ICD4D domain. SDS is supported by our mobile elicitation tool designed around the needs and capabilities of the targeted user population. We embed SDS in a requirements elicitation and specification process which commences with an initial domain analysis of the needs, goals, and tasks of the target population with respect to the high-level social development goals. The outcome of the preliminary domain analysis is used to formulate a set of questions for guiding the story narration. Once the stories are elicited, they are processed and the results are fed into a traditional requirements specification process. In this paper we propose a task-analytic approach for determining the topics of the guiding questions.
\end{abstract}

Keywords: Requirements Engineering, Requirements Elicitation, Structured Digital Storytelling, ICT4D.

\section{Introduction}

Requirements engineering is an important stage in any software development. It is more so in the case of software development for social development projects in rural areas of developing countries. Currently, a significant effort is being put into providing people in rural areas with access to digital content and services by using Information and Communication Technologies (ICT). Such projects, which seek to apply ICT in redressing socioeconomic inequalities, are referred to as "ICT for Development" or ICT4D. This article is concerned with requirements engineering in the ICT4D domain.

Unfortunately, often ICT4D projects fail because their development was driven by the technology available and not by the very needs and social problems of the people living in rural communities [1]. Existing technologies are often applied in a noninclusive manner with respect to the local population, without sufficient adaptation or re-invention, and often without regard for user's needs and their social contexts. 
Many of the failures are attributed to requirements miss-management [2] and could have been avoided if suitable requirements elicitation methods were applied.

It has been shown that traditional elicitation techniques assume that end users are able to understand and articulate their problems and needs in a reflective manner. Expressing oneself, with the right problem descriptions and the right needs, however, are all learnt skills, which because of their socioeconomic situation, people in rural communities may not have had the opportunity to develop [3]. An oral based mode of communication is more intuitive among such communities [4].

In order to address this shortcoming, we propose using storytelling as an alternative and complementary requirements elicitation technique. Storytelling is successfully applied in multiple domains [5;6], and is particularly suitable for people with limited literacy to express themselves and their needs. For the sake of eliciting requirements, we adapt the traditional storytelling concept with a task-oriented specialization and provide tool support, which leads to our notion of structured digital storytelling (SDS). With SDS, the narration is guided by a set of thematic questions. Before SDS can be applied, it is important to narrow the topic of the story to be told. For this purpose, a preliminary domain analysis is carried out determining needs, goals and tasks of the target population related to the project's high-level social development goals. Based on the task hierarchy a set of appropriate open-ended questions is selected and presented to the rural population. The elicitation phase is supported by a software tool (such as our E-Tool), which serves as a facilitator. The collected stories are analyzed and the resulting information is fed into a traditional top-down development process.

The remainder of this paper is structured as follows. The next section reviews relevant background information about requirements engineering and digital storytelling. Section 3 introduces the E-Tool for eliciting stories in a rural context. Then, in Section 4, we demonstrate how SRS can be embedded into a requirements elicitation and specification process. Section 5 discusses the case studies we have conducted. In Section 6, we discuss relevant related work. Finally, we conclude and provide an outlook to future work.

\section{Background}

In this section we remind the reader of the importance of proper requirements management and discuss key challenges when applied within an ICT4D context. We then describe key concepts of digital storytelling and discuss its advantages and disadvantages compared to traditional requirements elicitation techniques.

\subsection{Requirements Engineering in the ICT4D Domain}

Requirements engineering (RE) is a crucial step in the development of any software system. It is the process whereby the intended purpose of a system is discovered and documented so that it can be analyzed, communicated and eventually culminate in a software implementation that meets that purpose. How well that purpose is met is the primary measure of a system's success. Thus, RE is essential in determining what a system will do and how this will be measured. The process is inherently iterative, and consists of three major activities: elicitation of needs, requirements specification and 
requirements validation. The process starts with some ill-defined 'ideas' of what the system should do. These are elicited, analyzed, and systematically transformed into a technical requirements specification that defines the software system to be built completely and unequivocally.

The RE discipline offers a wide range of established methods and techniques for accomplishing the various activities, appropriate for different problem domains and development styles. The early involvement of end-users is a well-established principle of software engineering, with standard methods (such as interviews, workshops, focus groups and ethnographic studies) to facilitate the elicitation and communication of software requirements between stakeholders and analysts.

Eliciting software requirements in an ICT4D context poses a number of challenges due to the nature of the projects. Such projects target marginalized communities with the goal of assisting them in improving their socio-economic situation. The projects are driven by high-level social and economic development goals that almost invariably are initiated from outside the targeted community. Many involve multiple stakeholders such as social workers, agronomists, government and business representatives, etc. coming from the public, private, and non-profit sectors, and working in partnership to be the most effective. The intended beneficiaries typically have limited schooling, low literacy levels, and low disposable incomes while the developing regions and countries where the projects take place are characterized by inadequate infrastructures, intermittent power and connectivity, underdeveloped economic markets and distribution and support networks, and a lack of trained personnel.

\subsection{Structured Digital Storytelling}

Storytelling has been proven to be useful for people with limited literacy to express themselves and their needs. A recent study by Kerr [7] demonstrated the potential of digital storytelling for expressing community information, issues and frustrations; the very sources that will shed light on the real needs of users in rural areas. At its simplest, storytelling consists of someone telling their personal story on some topic and the narration being recorded. A facilitator may be present to ensure that items of interest are clarified and expanded. Recent digital technologies support the authoring of sophisticated multimedia stories that can be made accessible to a broad audience. Interactive Voice Response (IVR) systems that use structured dialogues to ask a sequence of questions, are an alternative approach for collecting user input..Our approach combines the two. We propose adding a multimedia, structured dialogue interface onto digital storytelling technology to assist people in expressing their information needs through stories which can then be shared in the community. Instead of asking direct questions about their information needs, the target population's needs can be elicited through a series of questions, short stories, 'what if scenarios', or by hearing their neighbors' views regarding the problems they face and their potential solutions. The structured dialogue ensures that relevant themes are covered, while hearing stories told by their neighbors will inspire people to tell their own stories.

In an ICT4D context, storytelling has a number of advantages over commonly used requirements elicitation techniques such as interviews, focus groups and ethnographic studies [8]. A major difference between SDS and the other elicitation techniques is that the narrator is largely left on their own to tell their story in their local language. 
Among the advantages we foresee with SDS is that storytelling capitalizes on the villagers' primary mode of communication. Although the questions provide some general guidance, an interviewer is not present to influence the narration. Thus, it is possible to identify problems and needs not initially envisaged and contextual factors that might otherwise be overlooked. Moreover, collecting stories involves fewer resources in terms of facilitators, preparation, and elapsed time, and it does not require facilitators who speak the local language.

A disadvantage with respect to other elicitation techniques is that there is no one present to provide clarifications, guidance or immediate follow-up on items of interest. Additionally, participants may focus their story on a single aspect leaving other equally relevant aspects unmentioned.

\section{The Mobile E-Tool}

In order to facilitate structured digital storytelling, we have developed a mobile tool, the E-Tool. It is a Java application and runs on cell phones with support for audio/voice recording. The motivation behind choosing a mobile elicitation device is the high penetration and ubiquitous availability of cell phones both in urban and rural contexts, especially in developing countries. For example, mobile phones have a $65 \%$ market penetration rate in India ${ }^{1}$. The technical requirements for the application are fulfilled by almost every low-budget cell phone available today.

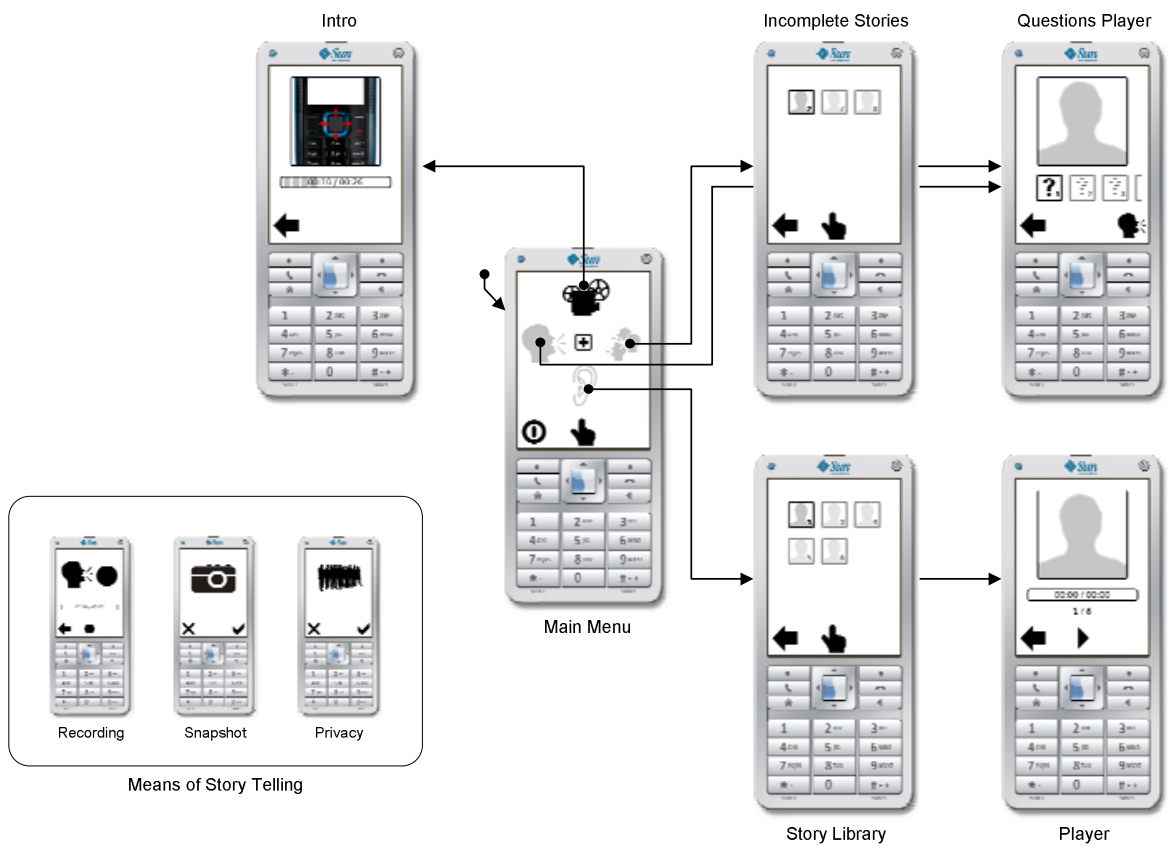

Fig. 1. Features and Navigation Structure of the E-Tool

\footnotetext{
${ }^{1}$ Telecom Regulatory Authority of India.
} 
The E-Tool is designed to be easy to use by a non-literate population. It provides (1) an introductory video explaining the reason for collecting the stories as well as an overview of how to operate the application, (2) a story library where the stories are stored and villagers can listen to them, and (3) an interview feature which guides users through a series of questions, letting them tell their own story. To keep the UI simple, it is based on a text-free design relying on iconic symbols, numbers from 0 to 9 , and audio prompting instead. Navigation is made quite simple by using symbols that are native to the specific community of people. Some of the features of the E-Tool as well as its navigation structure are portrayed in Figure 1.

While the absence of human facilitator bears the risk that the narrator will drift from the actual topic, we believe that without supervision the narrator has more liberty in determining the story's direction and thus put importance on things of his choice. Among the challenges to address, storytelling is by nature a social exchange that is enriched by the presence of an audience. As with any technique, consideration must also be given to confidentiality and self-censure, particularly when dealing with sensitive subjects. This can be achieved by providing each participating local community with only one cell phone at a time. The E-Tool offers the option to share stories in a public library or to keep them in a private space.

\section{SDS-Driven Requirements Elicitation and Specification Process}

In this section we detail a requirements management process based on the ideas and principles defined in the previous sections. That is, the process promotes the application of structured digital storytelling in an expanded context to elicit requirements from users. Our process is illustrated through a set of real-word models and artifacts stemming from a case study that conducted with farmers in rural India.

\subsection{Preliminary Analysis of Needs, Goals and Tasks}

$\mathrm{RE}$ is by nature difficult because it starts in a largely unconstrained problem space and much of the effort revolves around defining system boundaries, identifying relevant environmental conditions and prioritizing and selecting which requirements to pursue among the many possible options [9]. In the ICT4D domain requirements elicitation generally starts with determining what categories of information are vital to a rural society in some prioritized order. These categories identify the potential areas of intervention for a project, and invariably relate to a community's economic activity, and the health and welfare of its families.

In applying SDS, it is important to determine what topics to ask participants to speak about so that we can obtain a better understanding of their needs in the context of their activities, and thus bring out their specific concerns, issues and problems in situ. For this purpose, based on the high-level social development goals and with the assistance of domain experts, a preliminary domain analysis is carried out determining high-level user needs, goals and tasks. Needs are further decomposed into a set of goals. According to [10], a goal denotes a desired 'state' and is achieved by satisfying all of its sub-goals. Goals may support each other or be in conflict, and constraints will apply to certain goals. 
Under the premise of understanding the needs and goals of village farmers in rural India, we conducted an on-site domain analysis. The primary objective was to understand the root causes behind apparent problems and to relate them to a set of needs and goals. This comprised a detailed investigation of each contributing problem and a quantification of its individual impact. The analysis was carried out through a series of consultations and brainstorming sessions with domain experts as well as through observations and discussions with local farmers - the intended beneficiaries of the project.

Figure 2 portrays a subset of a needs-goal model. Farmers' needs are satisfied if the corresponding (a) farming, (b) financial, and (c) household goals are achieved. The farming goal is comprised of the goals associated with growing crops such as access to water and land, seed, fertilizers, pesticides and resources as well as the financing necessary to cover the significant upfront investment required to buy supplies, most often through loans. The financial goal is comprised of the goals arising from financing child education, parents' health care and farming activities. The household goal is comprised of the goals to educate children, care for aged parents and so forth.

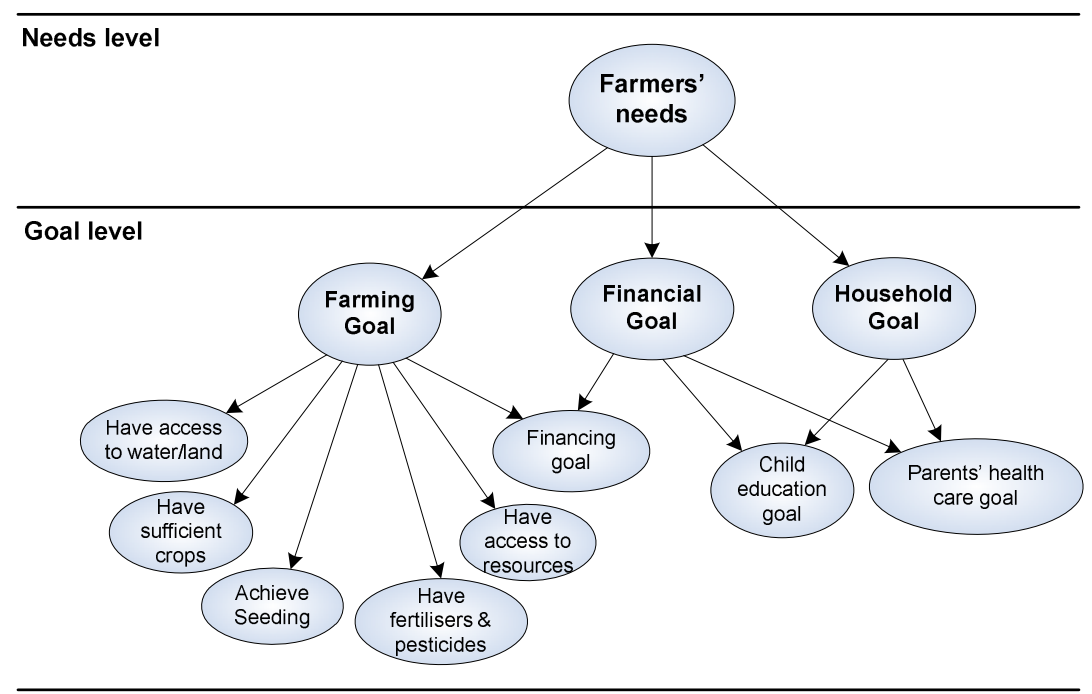

Fig. 2. Needs-goal model for Village Farmers in India

The needs-goal model is useful for representing the desires and concepts in the user's world; however it does not provide much insight into what activities they do. Therefore, as a next step, we associate each high-level goal with a task model that defines what tasks/activities are involved in attaining that goal. Most task modeling formalisms like HTA [11], GOMS [12], GTA [13], or CTT [14] make it possible to decompose tasks hierarchically into sub-tasks and to specify temporal constraints. 


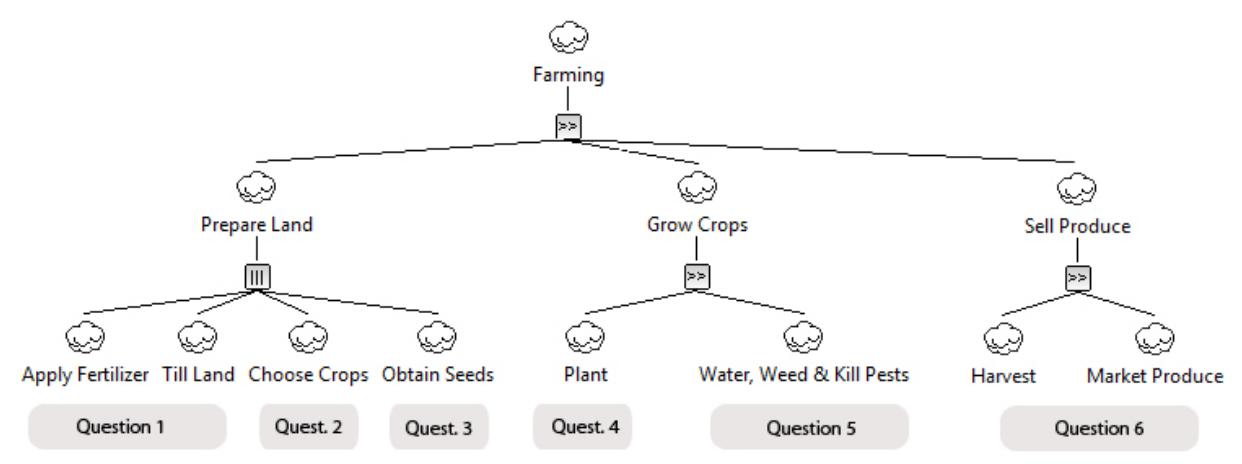

Fig. 3. Task Breakdown for Attaining the Farming Goal

The task-model depicted in Figure 3 provides a coarse-grained decomposition of the various activities necessary to attain the high-level goal of "Farming". In the example, the various subtasks are related by two temporal operators enabling (») and concurrency (III) denoting sequential composition and parallel composition, respectively. To farm, one must first prepare the land before one can grow crops and subsequently sell the produce. Preparing the land involves applying fertilizer, ploughing, choosing crops, and obtaining seeds. Growing crops includes the actual planting and the subsequent watering, weeding and elimination of pests; and finally, the sale of produce encompasses its harvesting and marketing.

\subsection{Question Selection and Requirements Elicitation}

Once a clear understanding of the high-level needs, goals and tasks of the intended beneficiaries has been obtained, a set of corresponding questions is formulated. The questions are chosen based on the tasks in the task model (as indicated in Figure 3) while the question context is obtained from the corresponding goals and sub-goals (Figure 2). By relating the questions to a particular context in which the task occurs, the questions will be closer to the farmers' actuality. Table 1 depicts a subset of the questions presented to village farmers in one of our case studies. Each question consists of a set of sub-questions and is suffixed by the request "Please tell us about such things" - inviting the participant to tell a comprehensive story.

Once the set of questions has been chosen, the collection of stories is relatively straightforward. An application (such as the E-Tool presented in the previous section) that plays the questions and records responses can be made available on a suitable mobile device in a location such as a community center, and villagers invited to try it. The various questions will be presented in the temporal order of the corresponding tasks so that the farmers are prompted to tell their story within a framework that is situated with respect to what they do. Participation can be encouraged by enlisting the support of respected members of the community and having them record their own stories to serve as examples in the story library. The time involved corresponds to the time it takes to tell the story, allowing a reasonable number of stories to be recorded in a few days. Because resources such as interviewers and facilitators are not involved, the application can readily be deployed in a number of villages, increasing the 
number of the stories collected and coverage of issues. Different topics can be addressed by changing the application prompts while different linguistic regions are easily supported by simply translating and rerecording the application prompts, making this elicitation method highly adaptable.

Table 1. List of Questions Presented to Village Farmers in Rural India

\begin{tabular}{|c|c|}
\hline Q1 & $\begin{array}{l}\text { "How many acres is your land? Do you own it or lease it? Do you have enough } \\
\text { water? How does water affect your yield? How do you prepare the land for } \\
\text { planting? Do you use fertiliser? Do you have difficulty getting it? Do you use } \\
\text { manual labor or machines? Is it costly or difficult? What if anything do you wish } \\
\text { to be better? Please speak about such things." }\end{array}$ \\
\hline Q2 & $\begin{array}{l}\text { "What are the different crops you cultivate? How many months for it to mature? } \\
\text { How much is the yield when it is the best? Do you cultivate always one type of } \\
\text { crop? If someone helps you to cultivate a different crop that can be more } \\
\text { profitable, will you try? What kinds of help will you need, like new seeds etc? } \\
\text { Please speak about such things." }\end{array}$ \\
\hline Q3 & $\begin{array}{l}\text { "How do you obtain the seeds for each crop? Do you buy or make your own? } \\
\text { How do you get good quality seeds? Please speak about such things." }\end{array}$ \\
\hline $\mathrm{Q} 4$ & $\begin{array}{l}\text { "To plant a crop, what expenses do you have? Do you have enough money or do } \\
\text { you have to borrow? If borrowing at what interest rate? Please speak about such } \\
\text { things." }\end{array}$ \\
\hline Q5 & $\begin{array}{l}\text { "Do you always have enough water for your crops? How does water affect your } \\
\text { yield? Tell us about the weeds and insects destroying or affecting your crop. How } \\
\text { do you tackle them? When there are too many insets, whom do you consult? How } \\
\text { do the pests affect your yield? Please speak about such things." }\end{array}$ \\
\hline Q6 & $\begin{array}{l}\text { "To harvest, do you use manual labor or machines? How much yield (bags or } \\
\text { kilos) do you get in a bounty crop? What do you do with the crop after harvest? } \\
\text { Where do you sell, in the 'mahndy' or to the local middle man? Where is the } \\
\text { price better? Do you have trouble to transport the produce? Please speak about } \\
\text { such things." }\end{array}$ \\
\hline
\end{tabular}

\subsection{Requirements Abstraction and Specification}

Following the elicitation phase, the stories collected are processed, analyzed and categorized. Since stories are told in people's local language, the first step after data collection consists of translating the stories into English. This is projected to be a manual activity, performed by local individuals (ideally from the same rural community) who possess an adequate degree of literacy in both the local language and English. Although relatively labor intensive, this process is straightforward and does not require highly skilled labor.

Following this, the transcriptions are analyzed to determine what issues the participants mention, their importance, and the various factors that play a role therein. It is here that a skilled qualitative analysis is required, to identify the various issues and related factors or conditions. For example, farmers complain of unfair pricing at the markets where they sell their produce. At the same time, farmers who have taken out loans are compelled to sell to the lender, thus bypassing the open market system. Any attempt to address the issue of unfair pricing must also consider how many farmers are constrained by loans and how they might benefit from a possible solution. Such 
issues are prioritized based on how critical they are and the frequency with which they are mentioned, and these along with the other factors are related back to the high-level goals. Additionally needs, domain concepts and desired features are abstracted as well. Due to the often large amount of information, we plan to employ manual as well as automated or semi-automated natural language processing to extract the information stated above. The applicability of corpus linguistics to document processing in requirements engineering has been shown in several problem domains and at different levels. E.g., Rolland and Proix [15] provide a general background for the applicability of natural language processing to requirements engineering.

Throughout the process of requirements production and refinement, statements from the different sources are integrated into a single set of requirements in the final software requirements specification (SRS). Finally, the SRS is validated to ensure that it reflects stakeholders' needs, and is sound and complete. The former is achieved by establishing traceability links to the user stories gathered during elicitation. It is ensured that each user need is addressed by at least one requirements statement and any unnecessary system features unrelated to any user needs are excluded. It is important that the requirements engineers remain in close contact to domain experts who will be able to reconcile differing goals and needs.

\section{Case Studies in Rural India}

In order to test the viability of SDS for requirements elicitation in an ICT4D context, we conducted three field studies in two rural areas of India using a prototype laptop version of the elicitation tool described in Section 3. In India there are wide disparities in socioeconomic conditions. Whereas the new economy centered on urban areas is giving rise to a comfortable middle class (and aspirations to those below), the traditional economy of the rural areas, largely based on farming, has left much of the rural population in extreme poverty with few options out [16;17].

We elicited stories on two different topics in two different rural regions with distinct regional languages. A total of three studies were conducted - two near Chennai in Tamil Nadu state and one near Bangalore in Karnataka state. All three took place in farming villages typical of the rural Indian context. In Tami Nadu, stories on farming and higher education were elicited while in Karnataka the topic was farming only. All together 30 stories were collected, 17 on farming and 13 on higher education. These were told by both male and female participants representing a broad age range, from children to the elderly, and a cross section of financial situations, from the very poor to those considered well off by local standards.

The acceptability of the SDS approach exceeded our expectations. All the participants were able to tell their story, and were enthusiastic about doing so. Villagers participated readily and quickly picked up the operation of the application. Once they began talking, they became engaged in telling their story and were not distracted by the mechanics of recording. While in almost all cases they participated in groups, their stories were highly personal and did not show any signs of "groupthink". At the same time, the group provided an audience for the teller, making the narration a natural communicative exchange. 
Our analysis of the stories indicated that they were highly useful in identifying and prioritizing the participants' concerns, and revealed an abundance of contextual information regarding their activities. While a discussion of this experiment is out of scope here, the results of our study are presented in [18]. Additionally, by conducting this experiment on different topics in different regions and languages, we demonstrated that this approach is both repeatable and readily adaptable to new contexts in terms of population group, language, and topic.

\section{Related Work}

In the case of ICT4D projects, the characteristics of the targeted user populations make it difficult to apply standard elicitation techniques. To date, although there is a growing body of research related to software development and deployment across national boundaries, little work has been done in the area of cross-cultural requirements gathering with stakeholders from disadvantaged socioeconomic backgrounds.

Over the past decade, although numerous ICT4D projects have been attempted, few have achieved long-term sustained success. A too great emphasis on technical success with inadequate consideration of end-users' needs and of the social development aspect of the projects are among the factors that have contributed to this lack of success [1]. The need to involve intended beneficiaries in determining project goals and constraints has long been recognized in social and economic development circles, and a number of participatory approaches such as Participatory Rural Assessment (PRA) based on the work of Chambers [19] exist for accomplishing this. However, such approaches are primarily geared towards identifying and introducing social and economic interventions and would need to be adapted to assist in determining software requirements. Furthermore, participatory approaches rely on techniques such as interviews, focus groups and community meetings, and thus encounter the same disadvantages as previously described when used for eliciting requirements in an ICT4D context.

In sociology, storytelling has proven itself as a means for developing a collaborative analysis from the bottom-up. In a recent study by Kerr [7] it was applied to identify the problems and constraints of homelessness as perceived by the homeless themselves. The resulting analysis revealed a number of significant issues that do not emerge from conventional top-down analyses where input is solicited from people such as social service providers, public officials and academic experts. There was a comparable divergence in the nature of potential solutions and associated issues as viewed from the top-down versus bottom-up. Furthermore, the research process of telling and listening to stories served as a catalyst to the homeless to become active in changing their situation.

In the ICT4D context, digital storytelling has been proposed as a means for sharing information among semi-literate people in rural villages. A study by Frohlich et al. [2] has shown the viability of storytelling as a means of communication in rural India. The study showed that villagers were enthusiastic about creating and listening to stories. Interestingly, the study also revealed a certain tension between those interested in creating and disseminating serious "development" content, and others more interested in creating personal and cultural content for entertainment purposes.

More recently, storytelling has been proposed as a means for eliciting requirements in domains where access to end-users and the actual context-of-use is restricted. In 
[6], the author describes a field study in the healthcare domain in which the requirements elicited using focus groups and interviews are compared to those elicited using focus groups and stories. The focus of this study was to determine any differences in the number, breadth and depth of themes addressed and the amount of time required by participants. The study concluded that there was no significant difference with respect to the number and breadth of themes addressed. However, storytelling and focus groups were more effective in eliciting more diverse context-of-use and social information, and storytelling required less time than interviews.

\section{Conclusion}

Due to the specific challenges that ICT4D projects present, the sole use of conventional requirements elicitation techniques is often insufficient. In this paper, we addressed this shortcoming and proposed structured digital storytelling (SDS) as a complementary technique for eliciting software requirements. Storytelling capitalizes on the primary mode of communication of the people living in rural areas. As such it is particularly suited for participants with low literacy. Compared to traditional elicitation techniques, the narrator is largely left on their own to tell their story in their local language. The use of an SDS approach does not exclude the use of other elicitation techniques. Indeed we see it as complementary, assisting stakeholders in validating that they are focusing on the right problem, and that the problem is thoroughly understood in the context in which it occurs.

SDS is facilitated by our mobile E-Tool, which allows for recording and playback of elicited stories. The tool is designed to be easy to use by a non-literate population and thus is equipped with a minimalistic, text-free user interface, which instead of text, uses graphical icons, buttons with distinct colors and audio prompts to identify and guide users through the various options. We have embedded SDS into a requirements management process, which starts off with an initial domain analysis phase during which high-level needs and goals of the rural population are elicited. Goals are further refined by a set of tasks performed to achieve the goals. Based on the various tasks a set of questions is selected which are used to configure the E-Tool. Once the stories are elicited, they will be processed and the information gained is fed into a traditional top- down requirements specification process.

We demonstrated utility of SDS in an ICT4D context by conducting three case studies in rural India using a prototype laptop version of the E-Tool. This will be followed by a field study whose aims are to validate the proposed requirements management process and to determine the usability of the mobile (cell-phone based) E-Tool. One challenge that remains to be addressed is the remote configuration and remote collection of the recorded voice clips.

\section{References}

[1] Heeks, R.: CT4D 2.0: The Next Phase of Applying ICT for International Development. IEEE Computer 41(6), 26-33 (2008)

[2] Frohlich, D.M., Rachovides, D., Riga, K., Bhat, R., Frank, M., Edirisinghe, E., Wickramanayaka, D., Jones, M., Harwood, W.: StoryBank: mobile digital storytelling in a development context. In: Proc. of Human Factors in Computing Systems, Boston, MA, USA, pp. 1761-1770. ACM, New York (2009) 
[3] Pitula, K., Radhakrishnan, T.: A Multimedia Tool to elicit Information Needs in Rural Communities. In: Proc. of HCI for Community and International Development Workshop at CHI 2008, Florence, Italy (2008)

[4] Ong, W.J.: Orality and literacy: The technologizing of the word. Routledge, New York (2002)

[5] Snowden, D.: Story telling: an old skill in a new context. Business Information Review 16(1), 30-37 (1999)

[6] Gausepohl, K.A.: Investigation of Storytelling as a Requirements Elicitation Method for Medical Devices, Masters Thesis in Industrial and Systems Engineering, Virginia Polytechnic Institute (2008)

[7] Kerr, D.: We Know What the Problem Is: Using Oral History to Develop a Collaborative Analysis of Homelessness from the Bottom Up. The Oral History Review 30(1), 27-45 (2003)

[8] Leffingwell, D., Widrig, D.: Managing software requirements: a use case approach. Addison-Wesley, Boston (2003)

[9] Cheng, B.H.C., Atlee, J.M.: Research Directions in Requirements Engineering. In: Proc. of Workshop on the Future of Software Engineering (ICSE 2007), pp. 285-303 (2007)

[10] Van Lamsweerde, A.: Goal-Oriented Requirements Engineering: A Guided Tour. In: Proc. of Fifth IEEE International Symposium on Requirements Engineering, pp. 149-163 (2001)

[11] Annett, J., Duncan, K.D.: Task Analysis and Training Design. Occupational Psychology 41, 211-221 (1967)

[12] Card, S., Moran, T.P., Newell, A.: The Psychology of Human Computer Interaction (1983)

[13] Veer, G., Lenting, B., Bergevoet, B.: GTA: Groupware Task Analysis - Modeling Complexity. Acta Psychologica 91, 297-332 (1996)

[14] Paternò, F.: Model-Based Design and Evaluation of Interactive Applications. Springer, Heidelberg (2000)

[15] Rolland, C., Proix, C.: A Natural Language Approach for Requirements Engineering. In: Loucopoulos, P. (ed.) CAiSE 1992. LNCS, vol. 593. Springer, Heidelberg (1992)

[16] Rezwan (2009). India: A wave of suicides among farmers. Global Voices Online (2009)

[17] Robinson-Sunna, S.: Seeds of Despair. Time.com (2007)

[18] Pitula, K., Radhakrishnan, T.: On Eliciting Requirements from End-Users in the ICT4D Domain. Submitted for Review to Journal of Requirements Engineering (2010)

[19] Chambers, R.: Rural Development: Putting the Last First. Prentice-Hall, Englewood Cliffs (1983) 